\title{
PRUEBAS EXPERIMENTALES DE EXTRACCION POR SOLVENTES PARA EL DISEÑO Y CONSTRUCCION DE MEZCLADORES - SEPARADORES A NIVEL DE LABORATORIO.
}

Francisco Gamarra G.' Francisco Rojas $\mathbf{T}^{2}$

\author{
RESUMEN
}

En el presente trabajo presentamos los resultados de las pruebas básicas que, junto con algunas consideraciones teóricas extraidas de la bibliografia, nos permitirán diseñar y construir mezcladores y separadores para la extracción por solventes, a nivel de laboratorio.

Se ha escogido una extracción, por formación de compuesto, en la cual el orgánico lo constituye el PT-5050 de la casa Acorga; y como acuoso, una solución de lixiviación de un mineral oxidado de cobre de Ite-Tacna.

\section{INTRODUCCION}

La extracción del cobre por solventes, es una técnica de purificación aplicada a soluciones pobres con la finalidad de concentrar el metal hasta un punto en que un proceso posterior, como la electrodeposición, pueda ser llevado a cabo.

La cinética es fundamental en el diseño de cualquier reactor, por lo que se consideró necesario estudiar tres aspectos: la cinética de extracción, de reextracción y la volocidad de separación de fases.

Como elemento acuoso se utilizó una solución proveniente de la lixiviación ácida de un mineral de cobre oxidado de Ite-Tacna. Esta solución, juntamente con el orgánico, se emplearon para realizar las pruebas necesarias que nos permitirán establecer el diseño de los

1. Ingeniero metalúrgico

2. Bachiller en metalurgia reactores y separadores en una planta de extracción por solventes, a nivel de laboratorio.

\section{MATERIALYMETODOS}

\section{Material}

Como orgánico se utilizó el PT-5050, y como acuoso la solución de lixiviación de un mineral de cobre oxidado de Ite-Tacna. Esta solución de lixiviación tuvo una concentración de 5,39 g/l de cobre y un $\mathrm{pH}=2,0$.

Además se utilizó querosene como diluyente.

Los equipos utilizados fueron:
a) Peras de decantación
b) Agitador magnético.
c) Vasos de precipitado
d) Potenciómetro.
e) Probetas. 
f) Soporte Universal.

g) Jeringas hipodérmicas.

h) Pipetas.

i) Cronómetro.

Todas las pruebas efectuadas fueron del tipo "batch".

\section{Métodos}

Las pruebas de cinética de extracción y re-extracción, fueron llevadas a cabo en condiciones ambientales, es decir, a temperatura de $23^{\circ} \mathrm{C}$ y a una atmósfera de presión.

\section{a) Cinética de extracción.}

Antes de iniciar las pruebas para determinar la cinética de extracción, se procedió a calcular la concentración del orgánico adecuado para nuestra solución de lixiviación.

El método consistió en dividir la concentración en cobre, dela solución de lixiviación, entre la capacidad de carga del orgánico:

$$
\begin{aligned}
& \% v=\frac{5,39 \mathrm{glCu}}{\%}=10,78 \\
& 0,50 \mathrm{~g} / \mathrm{l} / \% \mathrm{v}
\end{aligned}
$$

A esta concentración de orgánico se le dividió entre la capacidad de transferencia neta del orgánico que, según la práctica industrial, se encuentra entre el $65 \%$ a $75 \%$. Entonces:

$$
\% v=\frac{10,78}{0,75}=14,37
$$

Esta concentración del orgánico encontrada, se puede aproximar a $15 \% \mathrm{~V}$ como lea adecuada para nuestra solución de lixiviación.

Para determinar la cinética de extracción se mezcló a 900 rpm, el orgánico de concentración conocida con el acuoso, con una razón de fases O/Aigual auno. Durante el mezclado de las fases, se extrajeron muestras a los $30 \mathrm{seg}, 1 \mathrm{~min}, 2 \mathrm{~min}$, y $5 \mathrm{~min}$. Las muestras fueron analizadas para determinar el porcentaje de extracción de cobre.

\section{b) Cinética de re-extracción.}

Primeramente se cargó el orgánico de concentración conocida hasta su máxima capacidad, luego, éste se fue mezclando con una solución ácida sulfúrica de $150 \mathrm{~g} / \mathrm{l}$ de concentración. Durante el mezclado, las muestras son extraídas a los $30 \mathrm{seg}, 1 \mathrm{~min}, 2 \mathrm{~min}$ y $5 \mathrm{~min}$, y luego ser analizadas para determinar el porcentaje de re-extracción de cobre.

\section{c) Velocidad de separación.}

Para determinar la velocidad de separación de fases, se mezclóa 900 rpm, el orgánico de concentración conocida con el acuoso, con una razón de fases O/A igual a uno. Luego se transfirió rápidamentea una probeta de $250 \mathrm{~cm}^{3}$ decapacidad, en el cual se midió el tiempo de separación y la velocidad de separación de fases.

\section{RESULTADOS}

\section{a) Cinética de Extracción}

Se obtuvieron los siguientes resultados:

TABLA 1

\begin{tabular}{|c|c|}
\hline $\begin{array}{c}\text { Tiempo } \\
\text { (min) }\end{array}$ & $\begin{array}{c}\text { Extracción } \\
\text { (\%) }\end{array}$ \\
\hline 0,5 & 63,52 \\
1,0 & 92,09 \\
10,0 & 100,00 \\
\hline
\end{tabular}

\section{b) Cinética de Re-extracción}

\section{Se obtuvieron los siguientes}

TABLA 2

\begin{tabular}{|c|c|}
\hline $\begin{array}{c}\text { Tiempo } \\
\text { (min) }\end{array}$ & $\begin{array}{l}\text { Extracción } \\
\text { (\%) }\end{array}$ \\
\hline 1,0 & 91,25 \\
2,0 & 95,13 \\
10,0 & 100,00 \\
\hline
\end{tabular}

c) Velocidad de separación de fases

Se encontró que el tiempo que demoran las fases en separarse fue de 30 segundos y se midió una velocidad de separación de fases de 0,32 cm/s.

\section{DISCUSIÓN}

\section{a) Dimensionamiento del reactor y separador}

Se sabe que la cinética de una reacción química homogénea, es función de la concentración, temperatura, presióny del catalizador presente. Pero cuandola reacción química es heterogénea, es necesario considerar una variable más: el área de contacto. 
Sinosotros observamos las figuras 1 y 2 , veremos que mientras la diferencia de concentración, respecto al cobre, entre la fase acuosa y la fase orgánica, es menor, la cinética de extracción o re-extracción se volverá más lenta.

Como se habrá observado, la cinética de la reacción temperatura de $23^{\circ} \mathrm{C}$ ypresión, de una atmósfera aproximadamente, y no se ha creído conveniente cuantificar a la temperatura y la presión, sin embargo sabemos quelatemperatura destruye a cualquier reactivo orgánico. Por esa razón no hemos querido correr el riesgo de destruir a nuestro orgánico, aunque la temperatura pueda favorecer a la cinética de reacción. En cuanto a la presión, implementarla a nivel industrial sería impracticable por múltiples razones y además, no fue necesario cuantificarla, ya que la cinética de la reacción resultó buena. Finalmente respecto al área de contacto, ésta es controlada por la agitación, aquí fue necesario extraer la información de labibliografía técnica.

Por otro lado, se puede ajustar los datos

Figura 1

CINETICA DE EXTRACCION

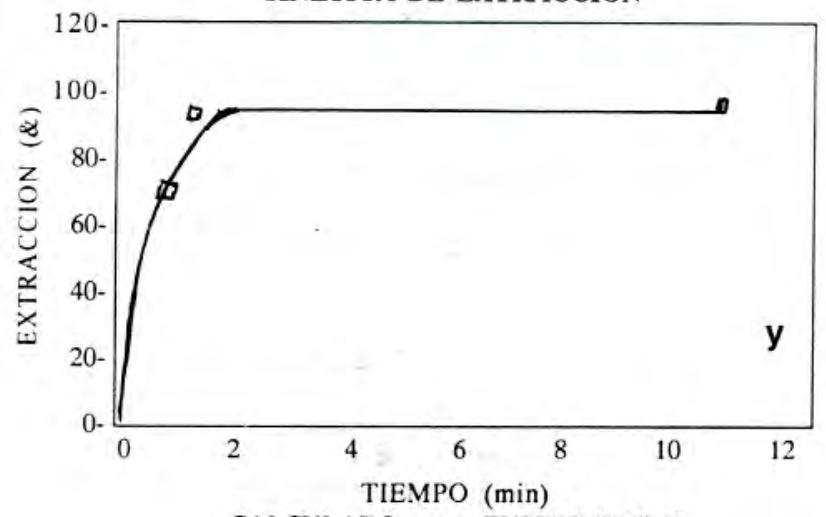

_CALCULADO O EXPERIMENTAL

Figura 2

CINETICA DE RE-EXTRACCION

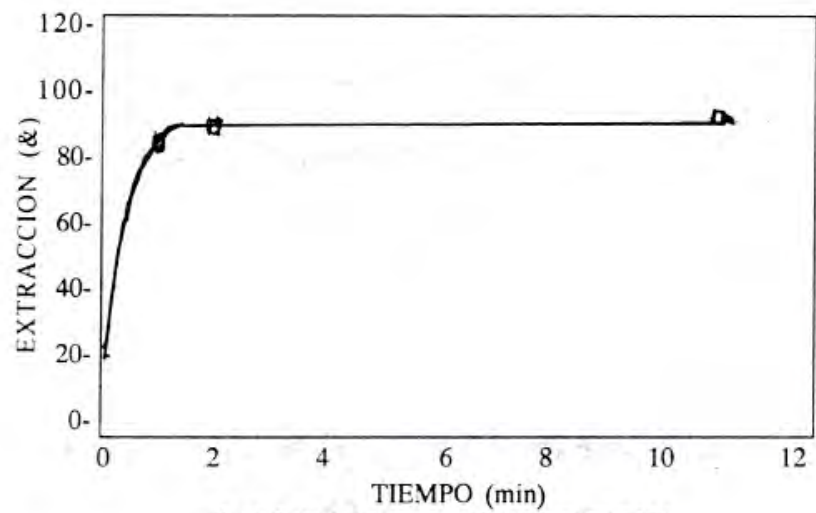

_CALCULADO O EXPERIMENTAL

experimentales obtenidos en las tablas 1 y 2 a una ecuación exponencial con parámetros. La ecuación para la extracóción sería:

$$
E(\%)=98.59\left[1-\exp ^{-2.235 t}\right]
$$

y para la re-extracción

$$
\begin{aligned}
& \operatorname{Re}-\operatorname{ext}(\%)=99.93\left[1-(1 / 10.945 t)\left(1-\exp ^{-10.945 t}\right)\right] \\
& \text { Las figuras } 1 \text { y } 2 \text { muestran los datos }
\end{aligned}
$$
experimentalescon los datos ajustados.

Podemos con todos estos datos dimensionar los reactores de extracción, y el de re-extracción. Si, por ejemplo, establecemos un flujo de $350 \mathrm{~cm}^{3} / \mathrm{min}$ y un tiempo de residencia de 2,0 minutos, tendremos que nuestro reactor debetener un volumen mojado de $700 \mathrm{~cm}^{3}$. Desdeluego, habráque darleun porcentaje de incremento, debido al volumen del agitador y al aumento de volumen cuando la mezcla es agitada.

En cuanto al tamaño del separador, éste puede ser calculado teniendo en cuenta la velocidad de separación. Si exageramos el tiempo de separación encontrado y lo establecemos en 1,5 minutos, y si además, lo relacionamos con el flujo de alimentación de $350 \mathrm{~cm}^{3} / \mathrm{min}$, tendremos que nuestro separador debe tener un volumen de $525 \mathrm{~cm}^{3}$. En función a este volumen, podremos calcular la altura de nuestra columna de separación fácilmente, con sólo establecer un área. Ahora, si establecemos una relación altura/diámetro, igual a 3,6 para la columna, podremos calcular su altura y su diámetro. Realizando los cálculos correspondientes, encontramos que la altura y el diámetro son de 20,54 y 5,70 centímetros respectivamente.

\section{b) Forma geométrica del reactor, separador y agitador}

En cuanto a la geometría del reactor, se tiene que ésta puede ser cilíndrica o cuadrada (figura 3). La ventaja de un reactor cuadrado radica en que no origina "vortex" y que además, son fáciles de construir, tanto a nivel de laboratorio como a nivel industrial. Reactores cilíndricos originan "vortex" y son más dificultosos cons truirlos a nivel industrial.

Figura 3

Tipo de mascladores

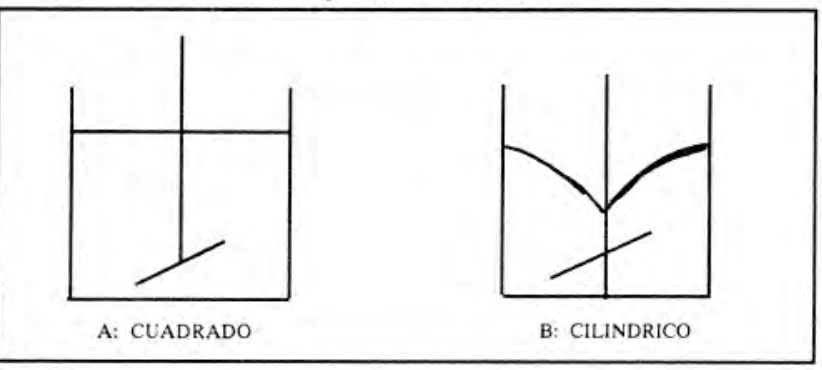

Respecto al equipo separador de fases, éste puede tener también dos formas: rectangular o cilíndrico (figura 4). Un separador cilíndrico a nivel de laboratorio es 
fácil de conseguir (por ejemplo tubos de PVC o fluorescentes) y la ventaja que tiene, a nivel industrial, es que ocupa menos área.

En cuanto a la forma del agitador existen numerosos diseños (figura 5). En cualquier caso, uno de ellos puede ser utilizado.

Figura 4

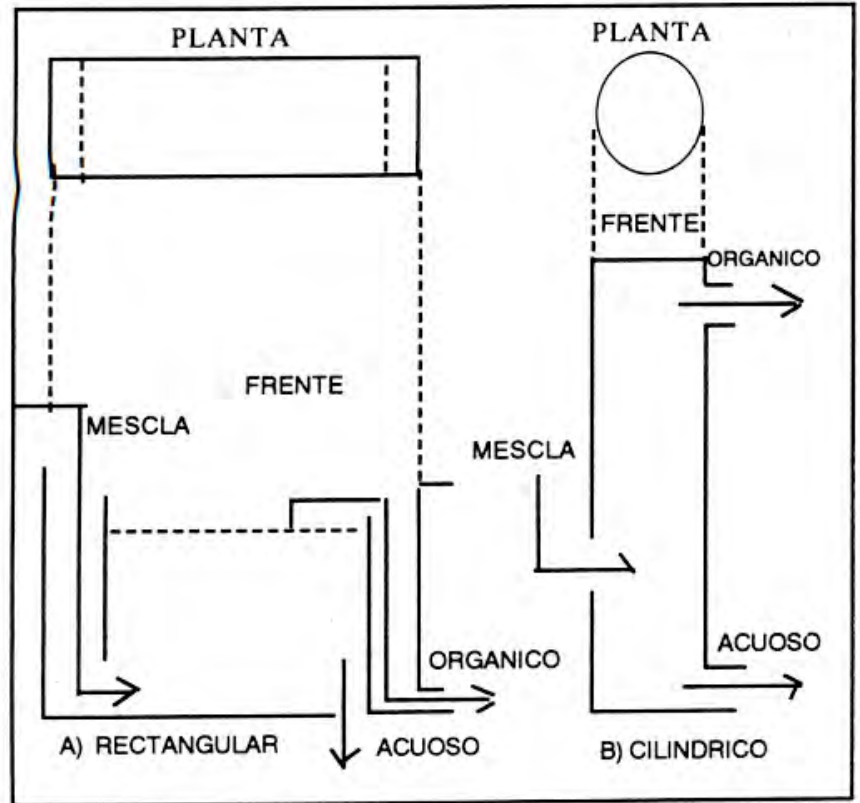

FIGURA 5

TIPOS DE AGITADORES

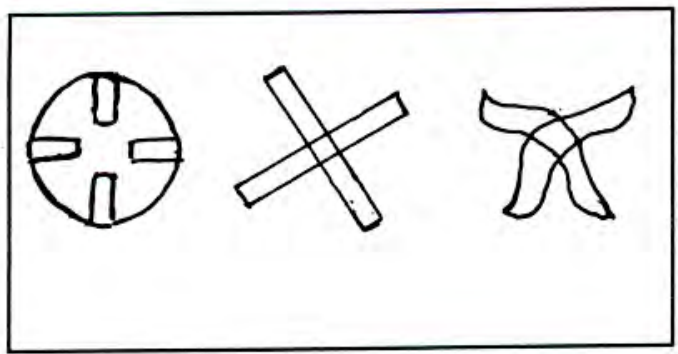

\section{c) Número de etapas.}

En cuando al número de etapas, varía según el metal de interés, y, por lo general, está entre 2-4 etapas, tanto en la extracción como en la re-extracción. En la figura 6 se puede observar una típica operación de extracción por solvente

FIGURA 6

\section{SISTEMA DE EXTRACCION POR SOLVENTES}

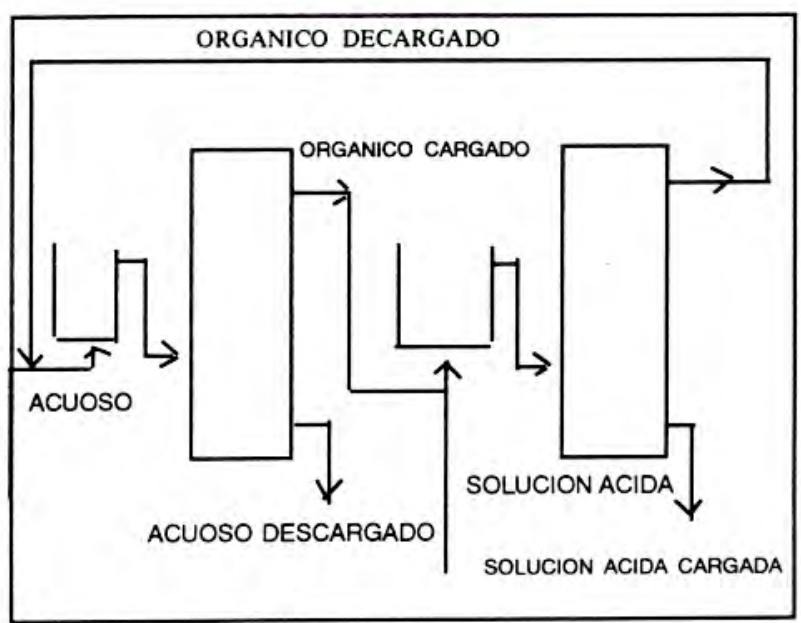

\section{CONCLUSIONES}

1. Es ventajoso construir un reactor de forma cuadrada para las etapas de extracción y re-extracción.

2.- Es ventajoso construir un separador de forma cilíndrica en primera instancia.

3. Se debe utilizar elementos de agitación y bombeo de por lo menos $1200 \mathrm{rpm}$, con sistema de control de rpm.

4.- Consideraciones en cuanto a la forma de alimentación, a los reactores, forma del agitador, altura del agitador, y otras consideraciones, tendrán que ser evaluadas con pruebas continuas.

\section{BIBUOGRAFA}

Lott, J.B. et al. 1972, Design of Large Scale MixerSttelers. By J.B.Lott, G.I.Warwich, and J.B. Schuffham, Transactions (AIME), vol.252, 27-35.

Revista Metal CENIN, 1980, Aplicaciones de la extracción con disolventes a la hidrometalurgia. Vol. 16, número 5 .
G.M. Ritcey. 1984, Solvent Extraction: Principles and appli cations to process matallurgy (Part I) Editorial Elsier-Amsterdam, páginas 68-72. 